\title{
On the Symmetry of the Gibbs States in Two Dimensional Lattice Systems
}

\author{
Charles-Edouard Pfister \\ Département de Mathématiques, Ecole Polytechnique Fédérale, \\ 61, Av. de Cour, Ch-1007 Lausanne, Suisse
}

\begin{abstract}
Under fairly general conditions if a two dimensional classical lattice system has an internal symmetry group $G$, which is a compact connected Lie group, then all Gibbs states are $G$-invariant.
\end{abstract}

\section{Introduction}

For a large class of classical lattice systems with an internal symmetry described by a continuous group $G$ all Gibbs states are also $G$-invariant if the space dimension is two $[1,2]$. One says that spontaneous symmetry breakdown is impossible. This phenomenon occurs in various other situations. We refer to [3] for examples and rigorous results in the field of statistical mechanics. Results of this kind are established here for classical lattice systems on $\mathbb{Z}^{2}$ with a compact connected Lie group $G$. A lattice system is given by a measure space $\Omega_{x}$, which is the space of configurations of the system at the lattice point $x$, a measure $d w_{x}$ on each $\Omega_{x}$ and a potential $U$ describing the interactions in the system. For example $\Omega_{x}=S^{1}$, the unit circle, $d w_{x}$ is the uniform measure on $S^{1}$ and $U$ is given by two-body interactions $-J(x-y) \cos \left(w_{x}-w_{y}\right)$ which are $G$-invariant with $G=S^{1}$ in an obvious way. Here $w_{x} \in \Omega_{x}$ and $w_{y} \in \Omega_{y}$. If $J(x-y)=|x-y|^{-\alpha}$, then the system is ferromagnetic. Theorem 1 below proves that for $\alpha \geqq 4$ all Gibbs states are $G$-invariant and there is no spontaneous magnetization. On the other hand if $2<\alpha<4$ there is spontaneous magnetization at low temperature and therefore there are Gibbs states which are not $G$-invariant [4]. This remains true with $\Omega_{x}=S^{n}$, the $n$-sphere in $\mathbb{R}^{n+1}$, and $\left(w_{x} \mid w_{y}\right)$ instead of $\cos \left(w_{x}-w_{y}\right)$, where $(-\mid-)$ is the Euclidean scalar product in $\mathbb{R}^{n+1} . G$ is $S^{n}$ and the results follow from [5] when $2<\alpha<4$.

The results of this paper extend previous results obtained by Dobrushin and Shlosman [1] and [2]. First of all the theorem below covers the case of power-law decaying interactions and not only exponentially decaying interactions (see also Remark 2 at the end of Sect. 2). This extension gives complete results for the class of ferromagnetic systems introduced above (see also Remark 1 at the end of 
Sect. 2). Finally the proof is quite different. It is based on a very simple physical argument dealing with the energy of configurations. This leads to a proof free of technical difficulties, which is not the case in [2]. Since the general case can be obtained from the case with $G=S^{1}$ (see [1]), the next section gives the results in this special situation and for two-body interactions only. Many-body interactions and the general case with a compact connected Lie group $G$ are treated in the last section.

\section{Main Results for $G=S^{1}$}

In this section $G=S^{1}$, the simplest compact connected Lie group, and the potential $U$ is given by two-body interactions $U_{x, y}$ only, $x$ and $y \in \mathbb{Z}^{2}$. For the sake of simplicity all $\Omega_{x}$ are the same and $U_{x, y}\left(w_{x}, w_{y}\right)=U_{x, y}\left(w_{y}, w_{x}\right)$ is translation invariant. The group $G$ acts on $\Omega_{x}$ and the action of $g \in G$ on $w_{x} \in \Omega$ is denoted by $g \cdot w_{x} \in \Omega_{x}$. The main assumptions are the following one.

a) $G$-Invariance. The measure $d w_{x}$ is $G$-invariant and the potential $U$ is $G$ invariant

$$
U_{x, y}\left(g \cdot w_{x}, g \cdot w_{y}\right)=U\left(w_{x}, w_{y}\right) .
$$

b) Smoothness. For any two-element subset of $\mathbb{Z}^{2}$, any fixed $w_{x}$ and $w_{y}$, the realvalued function

$$
g \rightarrow U_{x, y}\left(w_{x}, g \cdot w_{y}\right)
$$

defined on $S^{1}$ is twice differentiable. Since $S^{1} \cong \mathbb{R} / \mathbb{Z}$ this function may be considered as a periodic function on the real line $\mathbb{R}$. The first and second derivatives are denoted by $U_{x, y}^{\prime}$ and $U_{x, y}^{\prime \prime}$.

To express the decay property, which is the next condition, the following notations are used. If $x=\left(x^{1}, x^{2}\right) \in \mathbb{Z}^{2}$ then $|x|=\max \left(\left|x^{1}\right|,\left|x^{2}\right|\right)$. For each positive integer $k$ let

$$
f_{k}(\lambda)=\max \left(1, \ln _{k} \lambda\right)
$$

where $\ln _{k} \lambda=\ln \ln _{k-1} \lambda$ and let $f_{k}(\lambda)=1$ whenever $\ln _{k} \lambda$ is not defined. For $1<\beta<2 \quad f_{k}\left(\lambda^{\beta}\right) \leqq \beta f_{k}(\lambda)$.

c) Decay Property. Let

$$
J(|x-y|)=\left\|U_{x, y}^{\prime \prime}\right\|_{\infty}=\sup _{w_{x}, w_{y}}\left|U_{x, y}^{\prime \prime}\left(w_{x}, g \cdot w_{y}\right)\right| .
$$

There exist a positive constant $C$ and an integer $p$ so that

$$
\sum_{|y| \leqq L} J(|y|)|y|^{2} \leqq C \prod_{k=1}^{p} f_{k}(L) \equiv C F_{p}(L) .
$$

This means that the divergence of the above expression is at most like $\ln L \ln _{2} L \ldots$ $\ln _{p} L$ for large $L$.

Remark. It is also supposed of course that the Gibbs measures for finite systems 
are well-defined and so on. In particular

$$
\sum_{y}\left\|U_{x, y}\right\|_{\infty}<\infty
$$

in order that the thermodynamic limit makes sense.

Theorem 1. If a classical lattice system satisfies conditions $A, B$ and $C$, then all Gibbs states are G-invariant.

Proof. The proof is based on a physical intuitive argument of Herring an Kittel [6] showing, in the case of the ferromagnetic models described in the introduction, that there is no state with spontaneous magnetization.

Let $g \in G$ be fixed and let $\Lambda_{l}$ be the subset $\{x:|x| \leqq l\}$ of $\mathbb{Z}^{2}$. The main step in the proof is to show that for any configuration $w=\left(w_{x}, x \in \mathbb{Z}^{2}\right)$ of the infinite system there exists another configuration $\hat{w}=\left(\hat{w}_{x}, x \in \mathbb{Z}^{2}\right)$ with the properties

a) $\hat{w}_{x}=g \cdot w_{x}$, if $|x| \leqq l$

b) $\hat{w}_{x}=w_{x}$, if $|x| \geqq l+L$ for some $L$

c) $E(\hat{w})-E(w) \leqq K, K$ independent of $g$ and $l$

where $E(\hat{w})-E(w)$ is the energy difference between the two configurations. This quantity is well-defined since $w$ and $\hat{w}$ are different only over a finite region. Using the isomorphism between $G$ and $\mathbb{R} / \mathbb{Z}$ the identity element of $G$ is represented by 0 and the element $g$ by $\varphi \in[0,1)$ or by $\psi \in[-1,0)$ such that $\psi+1=\varphi$. Let

$$
0<\varphi_{L}<\varphi_{L-1}<\ldots<\varphi_{1} \leqq \varphi \text { and } 0>\psi_{L}>\psi_{L-1}>\ldots>\psi_{1} \geqq \psi .
$$

Each $\psi_{i}$ or $\varphi_{i}$ represents a well-defined element of $G$ denoted by the same symbol. The argument of Herring and Kittel suggests to define $\hat{w}$ as $w^{1}$ or $w^{2}$ where

$$
\begin{array}{lll}
w_{x}^{1}=w_{x} & w_{x}^{2}=w_{x}, & x \notin \Lambda_{L+l} \\
w_{x}^{1}=\varphi_{n} \cdot w_{x} & w_{x}^{2}=\psi_{n} \cdot w_{x}, & |x|=n+l, 1 \leqq n \leqq L \\
w_{x}^{1}=\varphi \cdot w_{x} & w_{x}^{2}=\psi \cdot w_{x}, & x \in \Lambda_{l} .
\end{array}
$$

In particular $\varphi \cdot w_{x}=\psi \cdot w_{x}=g \cdot w_{x}$. Let

$$
Q(L)=\sum_{1 \leqq k \leqq L} \frac{1}{k F_{p}(k)} .
$$

For large $L Q(L)$ diverges like $\ln _{p+1} L$.

The choice of $\varphi_{n}$ and $\psi_{n}$ is

$$
\varphi_{n}=\frac{\varphi}{Q(L)} \sum_{n \leqq k \leqq L} \frac{1}{k F_{p}(k)}
$$

and

$$
\psi_{n}=\frac{\psi}{Q(L)} \sum_{n \leqq k \leqq L} \frac{1}{k F_{p}(q)} .
$$

Therefore $\varphi_{1}=\varphi$ and $\psi_{1}=\varphi$. Let $\varphi_{x}$ (respectively $\psi_{x}$ ) be the rotation applied at $x$. 
For all $x$ and $y\left|\varphi_{x}-\varphi_{y}\right| \leqq 1$. For $l+1 \leqq|x|<|y| \leqq L+l$,

$$
\begin{aligned}
\varphi_{x}-\varphi_{y} & =\frac{\varphi}{Q(L)} \sum_{|x|-l \leqq k<|y|-l} \frac{1}{k F_{p}(k)} \\
& \leqq \frac{\varphi}{Q(L)} \frac{|x-y|}{(|x|-l) F_{p}(|x|-l)}
\end{aligned}
$$

Finally for $x \in \Lambda_{l}$ and $|y|>l$

$$
\varphi_{x}-\varphi_{y}=\frac{\varphi}{Q(L)} \sum_{1 \leqq k<|y|-l} \frac{1}{k F_{p}(k)} \leqq \frac{\varphi}{Q(L)} Q(|x-y|) .
$$

Similar estimates hold for $\psi_{x}$.

By hypothesis A

$$
U\left(w_{x}^{1}, w_{y}^{1}\right)=U\left(\varphi_{x} \cdot w_{x}, \varphi_{y} \cdot w_{y}\right)=U\left(w_{x},\left(\varphi_{y}-\varphi_{x}\right) \cdot w_{y}\right)
$$

By hypothesis B and with $\alpha \in(0,1)$

$$
U\left(w_{x}, \alpha \cdot w_{y}\right)=U\left(w_{x}, w_{y}\right)+U^{\prime}\left(w_{x}, w_{y}\right) \alpha+\frac{1}{2} U^{\prime \prime}\left(w_{x}, \theta \cdot w_{y}\right) \alpha^{2}
$$

for some $\theta$ depending on $w_{x}$ and $w_{y}, 0<\theta<\alpha$.

By hypothesis $\mathrm{C}$

$$
\sum_{y} J(|y|)|y|^{2-\varepsilon} \leqq C^{\prime}<\infty
$$

for fixed $\varepsilon>0$. Therefore

$$
\sum_{y} J(|y|) Q^{2}(|y|) \leqq C_{1}<\infty
$$

and there exists $\beta, 1<\beta<2$, such that

and

$$
\sum_{|y| \geqq L^{\beta}} J(|y|) Q^{2}(|y|) \leqq C_{2} L^{-3}
$$

$$
\sum_{|y| \leqq L^{\beta}} J(|y|)|y|^{2} \leqq C_{2} F_{p}(L)
$$

For a given $w$ the configuration $\hat{w}$ will be $w^{1}$ or $w^{2}$ according to the value of $E\left(w^{i}\right)-E(w)$.

$$
\begin{aligned}
E\left(w^{1}\right)-E(w)= & \sum_{x \in \Lambda_{L+1}} \sum_{y:|y|>|x|}\left\{U\left(w_{x}^{1}, w_{y}^{1}\right)-U\left(w_{x}, w_{y}\right)\right\} \\
= & \sum_{x \in \Lambda_{L+l}} \sum_{y:|y|>|x|} U^{\prime}\left(w_{x}, w_{y}\right)\left(\varphi_{y}-\varphi_{x}\right) \\
& +\sum_{x \in \Lambda_{L+l}} \sum_{\substack{y:|y|>l \\
|y|>l}} \frac{1}{2} U^{\prime \prime}\left(w_{x}, \theta \cdot w_{y}\right)\left(\varphi_{y}-\varphi_{x}\right)^{2} .
\end{aligned}
$$

The last line is smaller in absolute value than (see (2.1) and (2.2)) 


$$
\begin{aligned}
& \frac{1}{Q^{2}(L)} \sum_{x \in \Lambda_{l}} \sum_{y} J(|y|) Q^{2}(|y|)+\frac{1}{Q^{2}(L)} \sum_{x \in \Lambda_{L+l} \mid \Lambda_{l}} \sum_{|y| \leqq(|x|-l)^{\beta}} J(|y|) \frac{|y|^{2}}{(|x|-l)^{2} F_{p}^{2}(|x|-l)} \\
& +\frac{1}{Q^{2}(L)} \sum_{x \in \Lambda_{L+\ell \backslash \Lambda} l|y| \geqq(|x|-l)^{\beta}} J(|y|) Q^{2}(|y|) .
\end{aligned}
$$

By (2.3), (2.4) and (2.5) this is smaller than

$$
\begin{aligned}
& \frac{1}{Q^{2}(L)}(2 l+1)^{2} K_{1}+\frac{1}{Q^{2}(L)} C_{2} \sum_{n=1}^{L} 8(n+l) \frac{F_{p}(n)}{n^{2} F_{p}^{2}(n)} \\
& \quad+\frac{1}{Q^{2}(L)} C_{2} \sum_{n=1}^{L} 8(n+l) \frac{1}{n^{3}}=\frac{1}{Q^{2}(L)}\left(K_{1}(2 l+1)^{2}+K_{2} Q(L)+K_{3} l+K_{4}\right) .
\end{aligned}
$$

Therefore, for any fixed finite $l$, the last expression is smaller than some $K$, independent of $l$ and $g$ when $L$ is large enough. When $w^{1}$ is replaced by $w^{2}$ a similar estimate holds and the term containing the first derivatives of the potential is in this case

$$
\sum_{x \in A_{L+1} y} \sum_{\substack{|y|>|x| \\|y|>l}} U^{\prime}\left(w_{x}, w_{y}\right)\left(\psi_{y}-\psi_{x}\right)
$$

Since $\left(\psi_{y}-\psi_{x}\right)=\psi \varphi^{-1}\left(\varphi_{y}-\varphi_{x}\right)$ and $\psi \varphi^{-1}<0$ the above expression and the corresponding one for $w^{1}$ have different signs. Therefore for any configuration $w$ and any finite box $\Lambda_{l}$ and any rotation $g \in G=S^{1}$, there exists a configuration $\hat{w}$ such that

$$
E(\hat{w})-E(w) \leqq K
$$

This is the key estimate. The rest of the proof is an easy adaptation of the main result of [7].

Let $g \in G$ be given. Let $f$ be a positive local observable depending only on $w_{x}$ for $x \in \Lambda_{l}$. Let $\Lambda=\Lambda_{L+l}$ with $L$ large enough. The Gibbs state for the finite region $\Lambda$ and a fixed configuration $w_{\Lambda^{c}}$ outside $\Lambda$ is given as usual by the measure on $\prod_{x \in \Lambda} \Omega_{x}=\Omega^{\Lambda}$

$$
v_{\Lambda}\left(d \eta_{\Lambda} \mid w_{\Lambda^{c}}\right)=\frac{\exp \left(-E\left(\eta_{\Lambda} \mid w_{\Lambda^{c}}\right)\right) d \eta_{\Lambda}}{Z_{\Lambda}\left(w_{\Lambda^{c}}\right)} .
$$

Let $\mu$ be any Gibbs state of the infinite system and $\mu_{g}$ the Gibbs state obtained from $\mu$ by a rotation $g$. By definition of Gibbs state the expectation value of $f$ in the state $\mu_{g}$ is

$$
\langle f\rangle_{\mu_{g}}=\int \mu(d w) f(g \cdot w)=\int \mu(d w) \int v_{\Lambda}\left(d \eta_{\Lambda} \mid w_{\Lambda^{c}}\right) f\left(g \cdot \eta_{\Lambda}\right) .
$$


Let $w_{A c}$ be fixed. On $\Omega^{A}$ the transformations $T_{1}$ and $T_{2}$ are one-to-one

$$
\begin{aligned}
& T_{1}: w_{\Lambda} \mapsto w_{A}^{1} \\
& T_{2}: w_{A} \mapsto w_{A}^{2}
\end{aligned}
$$

where $w_{A}^{1}$ and $w_{A}^{2}$ are the configurations studied before. They leave the measure $d \eta_{A}$ invariant by hypothesis A. Furthermore they coincide with the rotation $g$ on $\prod_{x \in A_{l}} \Omega_{x}$.

Finally there exists a partition of $\Omega^{4}$ in two subsets $\Omega_{1}$ and $\Omega_{2}$ such that

$$
E\left(T_{i} w_{A}\right)-E\left(w_{A}\right) \leqq K, \forall w_{\Lambda} \in \Omega_{i} .
$$

Consequently if $\chi_{i}$ is the characteristic function of $\Omega_{i}$

$$
\begin{aligned}
& Z_{\Lambda}\left(w_{A^{c}}\right) \int v_{\Lambda}\left(d \eta_{\Lambda} \mid w_{A^{c}}\right) f\left(g \cdot \eta_{\Lambda}\right)=\sum_{i=1,2} \int d \eta_{\Lambda} \exp \left(-E\left(\eta_{\Lambda} \mid w_{A^{c}}\right)\right) \chi_{i}\left(\eta_{\Lambda}\right) f\left(T_{i} \cdot \eta_{\Lambda}\right) \\
& =\sum_{i=1,2} \int d \eta_{\Lambda} \exp \left(-E\left(T_{i} \cdot \eta_{\Lambda} \mid w_{\Lambda^{c}}\right)\right) \chi_{i}\left(\eta_{A}\right) f\left(T_{i} \eta_{\Lambda}\right) \\
& \cdot \exp \left(-E\left(\eta_{\Lambda} \mid w_{A^{c}}\right)+E\left(T_{i} \cdot \eta_{\Lambda} \mid w_{A^{c}}\right)\right) \\
& \leqq e^{K} \sum_{i=1,2} \int d \eta_{\Lambda} \exp \left(-E\left(T_{i} \cdot \eta_{\Lambda} \mid w_{A^{c}}\right)\right) \chi_{i}\left(\eta_{A}\right) f\left(T_{i} \cdot \eta_{A}\right) \\
& \leqq 2 \cdot e^{K} \int d \eta_{\Lambda} \exp \left(-E\left(T_{i} \cdot \eta_{\Lambda} \mid w_{A^{c}}\right)\right) f\left(T_{i} \cdot \eta_{A}\right) \\
& =2 \cdot e^{K} \int d \eta_{\Lambda} \exp \left(-E\left(\eta_{\Lambda} \mid w_{A^{c}}\right)\right) f\left(\eta_{\Lambda}\right) \text {. }
\end{aligned}
$$

Using this inequality in (2-6) and integrating with respect to $\mu$ gives

$$
\langle f\rangle_{\mu_{g}} \leqq \tilde{K}\langle f\rangle_{\mu}
$$

where $\tilde{K}$ is independent of $f, \mu$ and $g$. Therefore there exists $0<\tilde{K}<\infty$ independent of $f, \mu$ and $g$ such that

$$
\tilde{K}^{-1}\langle f\rangle_{\mu_{g}} \leqq\langle f\rangle_{\mu} \leqq \tilde{K}\langle f\rangle_{\mu_{g}} .
$$

Since these last inequalities are true for the characteristic functions of cylindrical subsets, they remain true, by a limiting procedure, for any characteristic functions of subsets of the tail field. Let $\mu$ be an extremal Gibbs state. These inequalities show that $\mu$ and $\mu_{g}$ coincide on the tail field (since $\mu_{g}$ is extremal) and therefore $\mu=\mu_{g}$ i.e. $\mu$ is $g$ invariant for all $g \in G$. This finishes the proof.

Remark 1. The example of ferromagnetic models described in the introduction shows that the theorem is valid if $\alpha \geqq 4$. It is not valid if $\alpha<4$. More precisely the theorem is valid for the coupling constants $J(|x-y|)$ behaving for large $|x-y|$ like

$$
(|x-y|)^{-4} \ln _{2}|x-y| \ldots \ln _{p}|x-y| .
$$

On the other hand the proof fails if the behavior of $J(|x-y|)$ for large $|x-y|$ is like

or even

$$
(|x-y|)^{-4}(\ln |x-y|)^{\varepsilon}
$$

$$
(|x-y|)^{-4} \ln _{2}|x-y| \ldots \ln _{p-1}(|x-y|)\left(\ln _{p}(|x-y|)\right)^{1+\varepsilon}
$$


with $\varepsilon>0$. In fact the theorem is not true in these cases. Indeed using the results of [5] it is sufficient to find a reflection positive potential with such a behavior for large $|x-y|$ in order to have a counter-example to the theorem. Potentials with such a behavior can be constructed [9].

Remark 2. If the interactions $U_{x, y}$ have an exponential decay for large $|x-y|$, then the condition $C$ can be weakened and the growth condition $f_{1}(L) \ldots f_{p}(L)$ replaced by $L$. One uses the exponential decay as follows.

Let

$\left\|U_{x, y}\right\|_{\infty} \leqq c e^{-\kappa|x-y|}$. Then there exists $\beta>0$ such that

$$
\sum_{\substack{y: \\|y-x| \geqq \ln L^{\beta}}}\left\|U_{x, y}\right\|_{\infty} \leqq \frac{1}{L^{3}} .
$$

Therefore it is sufficient to be able to bound the sum with $U_{x, y}^{\prime \prime}$ only for $|x-y| \leqq \ln L^{\beta}$. This is possible if $\varphi_{n}=\frac{\varphi}{Q(L)} \sum_{k \geqq n}^{L} \frac{1}{k}$ and $Q(L)=\sum_{k=1}^{L} \frac{1}{k}$.

Therefore the results of Shlosman [2] are covered.

Remark 3. The results are still valid for systems extended in three dimensions provided the thickness is finite.

Remark 4. No particular property of the space $\Omega_{x}$ of the configurations at $x$ is used in the proof. However since the condition $C$ is expressed through the sup-norm $\|\cdot\|_{\infty}$ genuine models of unbounded spin systems in statistical mechanics do not satisfy the hypothesis of the theorem.

Remark 5. Examples of systems with a continuous symmetry group and with several phases in two dimensions can be found in the work of Shlosman [8].

Remark 6. It is sufficient to have that $U\left(w_{x}, \alpha \cdot w_{y}\right)=U\left(w_{x}, w_{y}\right)+U^{\prime}\left(w_{x}, w_{y}\right) \alpha+$ $\theta_{x, y}\left(w_{x}, w_{y}, \alpha\right) \cdot \alpha^{2}$ with $\left|\theta_{x, y}\left(w_{x}, w_{y}, \alpha\right)\right| \leqq J(|x-y|)$, where $\theta_{x, y}\left(w_{x}, w_{y}, \alpha\right)$ is some real valued function. Therefore if $U_{x, y}^{\prime}$ exists and satisfies a kind of Lipschitz condition the theorem is also valid.

Remark 7. Concerning the idea, at the end of the proof, which was taken in [7], one should mention earlier works of Sakai in [10] and of Rost, reported in [11].

\section{Generalizations}

\subsection{Many Body Interactions}

The restriction to two body interactions can be removed. In the general case the potential $U$ is a family of functions $U_{A}$ indexed by the finite subsets $A$ of $\mathbb{Z}^{2}$. Let $A$ be the subset $\left\{x_{1}, \ldots, x_{n}\right\} \subset \mathbb{Z}^{2}$. The function $U_{A}$ is defined on $\Omega^{A}=\prod_{x \in A} \Omega_{x}$ and an element of $\Omega^{A}$ is $w_{A}=\left(w_{x}: x \in A\right)$. Let $U_{A}$ be a symmetric function of its arguments $w_{x_{1}}, \ldots, w_{x_{n}}$. Let $\varphi$ be a rotation. The function $U_{A}$ must be $G$-invariant:

$$
U_{A}\left(\varphi \cdot w_{x_{1}}, \ldots, \varphi \cdot w_{x_{n}}\right)=U_{A}\left(w_{x_{1}}, \ldots, w_{x_{n}}\right) .
$$


For fixed $w_{A}$ and for $\alpha_{2}, \ldots, \alpha_{n} \in G, U_{A}\left(w_{x_{1}}, \alpha_{2} \cdot w_{x_{2}}, \ldots, \alpha_{n} \cdot w_{x_{n}}\right)$ defines a function on $G \times \ldots \times G(n-1$ factors $)$. The function must be twice differentiable in the variables $\alpha_{2}, \ldots, \alpha_{n}$.

Let

$$
J(A)=\sum_{j=2}^{n} \sum_{k=2}^{n}\left\|U_{A, \alpha_{j}, \alpha_{k}}\right\|_{\infty}\left|x_{1}-x_{j}\right|\left|x_{1}-x_{k}\right|
$$

where $U_{A, \alpha_{j} \alpha_{k}}$ is the derivative of $U_{A}$ with respect to $\alpha_{j}$ and $\alpha_{k}$. Then the decay condition becomes

$$
\sum_{\substack{A \ni x_{1} \\ \Lambda_{L}\left(x_{1}\right) \cap\left(A \backslash\left\{x_{1}\right\}\right) \neq \varnothing}} J(A) \leqq C \prod_{i=1}^{p} f_{i}(L)
$$

with $\Lambda_{L}\left(x_{1}\right)=\left\{x:\left|x-x_{1}\right| \leqq L\right\}$. Under these conditions theorem 1 is still valid.

\subsection{Compact Connected Lie Groups}

An argument used by Dobrushin and Shlosman [1] shows that the case where $G$ is a compact connected Lie group follows from the previous situation. Smoothness and decay conditions are as before. The reduction of the general case to the case $G=S^{1}$ is done as follows. For any element $g \in G$ there is a one parameter subgroup of $G$ containing $g$. If this subgroup is closed then it is isomorphic to $S^{1}$. Otherwise the closure of this subgroup is isomorphic to a torus. This shows that there exists a dense subset $G_{0}$ of $G$ such that any element of $G_{0}$ is contained in a subgroup isomorphic to $S^{1}$. From the proof of the theorem it is clear that in the general case this is sufficient in order to prove Theorem 1 under the appropriate smoothness and decay conditions.

Acknowledgements. I thank C. Gruber, H. Kunz and T. Spencer for very useful conversations and comments and H. Georgii. I thank B. Simon for correspondence, in particular for his comments on Remark 1 and [10].

\section{References}

1. Dobrushin, R. L., Shlosman, S. B. : Commun. Math. Phys. 42, 31 (1975)

2. Shlosman, S. B. : Teor. Mat. Fiz. 33, 86 (1977)

3. Mermin, N. D. : J. Phys. Soc. Jpn 26, Suppl. 203 (1969)

4. Kunz, H., Pfister, C. E.: Commun. Math. Phys. 46, 245 (1976)

5. Fröhlich, J., Israel R., Lieb, E. H., Simon, B.: Commun. Math. Phys. 62, 1 (1978)

6. Herring, C. H., Kittel, C.: Phys. Rev. 81, 869 (1951)

7. Bricmont, J., Lebowitz, J. L., Pfister, C. E.: J. Stat. Phys. 21, 573 (1979)

8. Shlosman, S. B.: Commun. Math. Phys. 71, 207 (1980)

9. Simon, B.: private communication

10. Sakai, S.: Commun. Math. Phys. 43, 39 (1975); J. Funct. Anal. 21, 203 (1976); Tóhoku Math. J. 28, 583 (1976)

11. Georgii, H. O.:Théorie ergodique Rennes 1973/74. In: Lecture notes in mathematics, Vol. 532 p. 532 Berlin, Heidelberg, New York: Springer 1976

Communicated by E. Lieb

Received July 29, 1980 